\title{
Millimeter Wave Beam Steered Fiber Wireless Systems for 5G Indoor Coverage: Integrated Circuits and Systems
}

\author{
(Invited Talk)
Zizheng Cao ${ }^{1}$, Xuebing Zhang ${ }^{1}$, Xinran Zhao ${ }^{1}$, Longfei Shen ${ }^{1}$, Xiong Deng ${ }^{1}$ Xin Yin ${ }^{2}$ and Ton Koonen ${ }^{1}$
${ }^{1}$ Institute for Photonic Integration, Eindhoven University of Technology, PO Box 513, 5600MB Eindhoven, the Netherlands
${ }^{2}$ Ghent University, Ghent, Belgium
Author e-mail address: z.cao@tue.nl

\begin{abstract}
In this talk, we review our recent progress and on-going research on millimeter wave beam steered fiber wireless systems for 5G indoor coverage enabled by the advanced photonic integrated circuit and well-designed fiber-wireless networks.

OCIS codes: (130.3120) Integrated optics devices; (060.5625) Radio frequency photonics; (060.2330) Fiber optics communications
\end{abstract}

\section{Introduction}

The explosion of communication traffic is required by an insatiable appetite for high speed internet connectivity and video-based content delivery to indoor wireless and mobile terminal users. The next generation wireless networks (5G) are expected to provide 1000 times higher wireless area capacity in 2020 compared to the one in 2010 [1]. To boost the capacity in the spectral domain, the millimeter wave (mm-wave) communication is a promising solution by exploring the huge bandwidth at higher frequency [2, 3]. There are two challenges for mm-wave communications: one is the high penetration loss through walls, which makes inter-room connection/coverage difficult; the other is the small electrical aperture induced low antenna gain. Fiber wireless systems with loss-less fiber links and multiple simplified remote antenna sites can overcome the inter-room connection/coverage problem, while the issue of low antenna gain can be overcome by beam steering (and beamforming) techniques. Samsung recently proposed and demonstrated a mm-wave communication empowered by beam steering [4], which is based on electronic devices. For broadband beam steering, true time delay (TTD) is required. However, the electronic integrated circuits (for instance (Bi)CMOS ) suffer from high loss at high frequencies. Optical true time delay (OTTD) with inherent low loss and broad bandwidth can be used for broadband mm-wave beam steering to avoid the beam squint problems [5-7]. Fiber wireless systems incorporating OTTD-based radio beam steering have been proposed for indoor networks [8]. In such work, OTTD is beneficial to the radio over fiber (RoF) systems since no additional electrical-optical and optical-electrical conversion is required. However, the optical tunable delay lines (OTDLs) employed in [8] are based on bulk-optics components, which limit their further applications. The integrated OTDLs are of demand [9] for compact and stable optical mm-wave beam steering. Recently, we designed and characterized a remotely tunable integrated OTDL based on an arrayed waveguide grating feedback loop (AWG-loop). Since the AWG is used as both the wavelength multiplexer (MUX) and the de-multiplexer (de-MUX), the AWG-loop allows a compact, fabrication tolerant and scalable solution for integrated OTDL [10]. Thanks to this AWG-loop, a series of advanced system features are guaranteed: 1) the antenna sites can be simplified with a single remotely tunable OTDL chip without active control; 2) multiple beams supporting multiple users can be realized simultaneously due to its linear feature; 3 ) it is low cost, compact and power efficient compared with its discrete component counterpart.

\section{Photonic integrated optical beam-former}

The proposed integrated AWG-loop and its corresponding AWG-loop delay network (ALDN) are shown in Fig. 1 to serve as a photonic mm-wave beam-former. The 1-by-2 ALDN comprises an optical power splitter and an AWGloop. The RoF signal is split into two paths: one goes to the first output directly (Output-1) and the other passes the AWG-loop till the second output (Output-2). As shown in Fig. 1(a), the AWG-loop is realized by an N-by-N spectral-cyclic AWG. It is used as both the wavelength MUX and the de-MUX. The AWG, which is cyclic in optical spectrum, allows further interleaved operation for 2-D optical mm-wave beam steering [5]. The feedback loops (optical waveguides) link N-1 pairs of inputs and outputs of the AWG symmetrically for re-circulating operations. One pair of input and output is left out as the input and output of the entire device (AWG-loop). This AWG-loop works as a stepwise OTDL. It is topologically equivalent to two AWGs in series with a delay element in between. As shown in Fig. 1(b), the delay of Output-2 is determined by the wavelength of the signal. The delay of 
(a) AWG-loop delay network
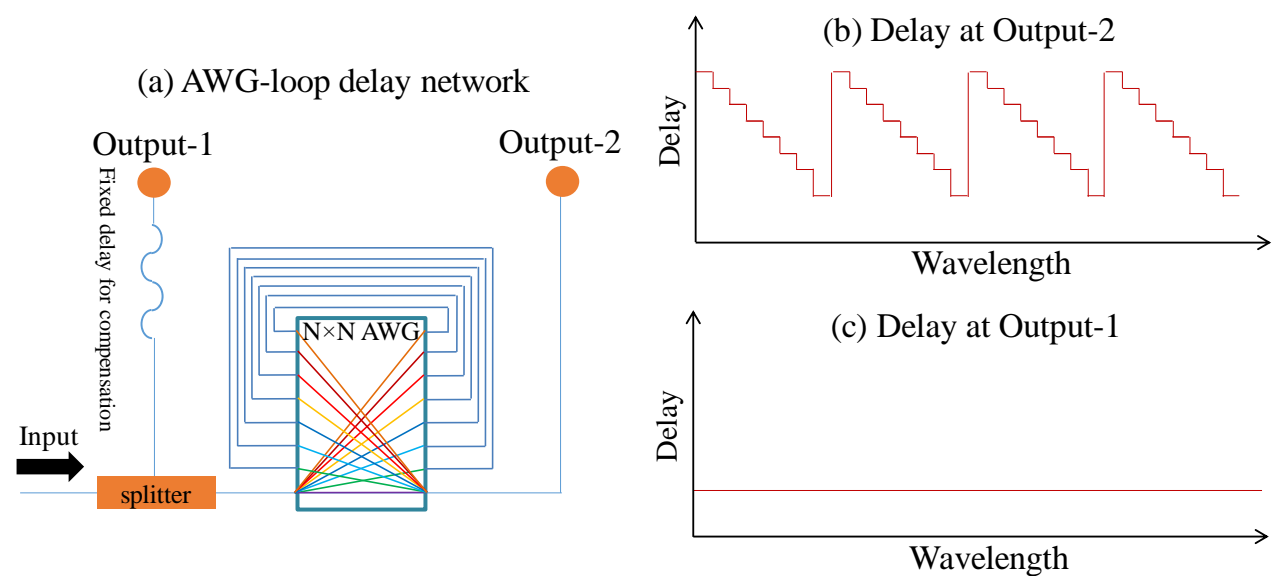

Fig.1.The principle of AWG-loop based integrated optical tunable delay line (OTDL), (a) AWG-loop delay network; (b) delay at Output-2 of (a), (c) delay at Output-1 of (a).

Output-1 is schematically shown in Fig. 1(c). A compensation optical delay line is employed to avoid initial delay offset between Output-1/-2. By re-using the same AWG as MUX and de-MUX, the footprint can be significantly reduced (approximately by half) and the relative spectral mis-alignment between the MUX and de-MUX vanishes. More experimental details regarding the AWG-loop will be present in this talk.

\section{System architecture}

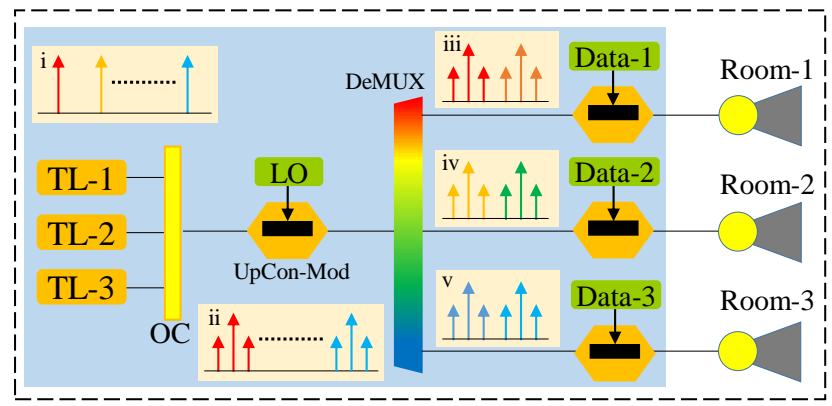

(a) Low density star architecture

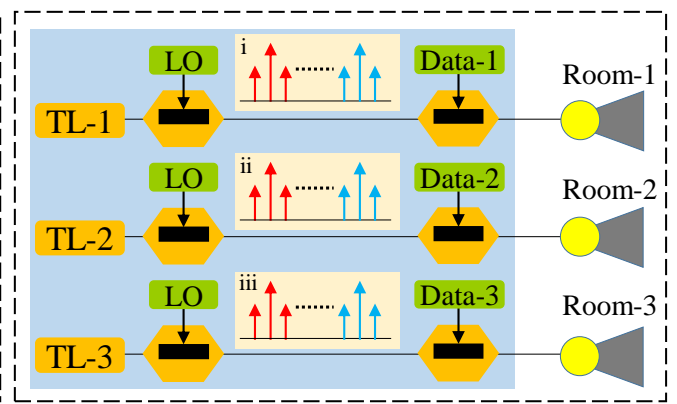

(b) High density P-t-P architecture

Fig.2. The mm-wave beam steered fiber wireless system for indoor coverage. (a) star network architecture; (b) the point-to-point (P-t-P) network architecture. TL: tunable laser; OC: optical combiner; LO: local oscillator.

The AWG-loop is, in essence, a step-wise wavelength-tuned discrete delay unit. The step number of tuned beams in an AWG-loop is equal to the number of tuned wavelengths. To get rid of cross talk, there is a minimal value for the spectral spacing between each wavelength. Therefore, the spectral range of the optical mm-wave signal defines the upper boundary of the available beam number (beam density). Here two network architectures are proposed: one is the star architecture for low density of tuned beams; the other is the point-to-point (P-t-P) architecture for high density of tuned beams. The low density architecture is designed for low capacity systems with low complexity while the high density one aims for high capacity systems.

As shown in Fig. 2 (a), all the wavelengths generated from tunable lasers (TL-1/2/3) in the star architecture are combined. The spectrum is schematically shown in inset $\mathrm{i}$. Different colors denote different channels (at different wavelengths). The multi-channel signal is then modulated by a local oscillator (LO) through an up-conversion modulator (UpCon-Mod) with the spectrum shown in inset ii. Afterwards, the wavelengths are separated into multiple wavelength groups by a wavelength de-MUX for routing to different rooms. The spectra after de-MUX are shown in inset iii/iv/v. Each wavelength group carries different services for each room. The beam density in a room is determined by the wavelength tuning range within its allocated wavelength group. Since the whole spectral range is divided into many pieces for different rooms, the beam density of each room is low. As shown in Fig. 2 (b), the high density P-t-P architecture is consist of separate fiber channels for each room. It means that the whole spectral range can be fully used for each room, as shown in inset i/ii/iii, resulting in a high beam density. However, for each room, separate up-conversion modulators are employed. The advantage of the high density architecture is achieved 
with the sacrifice of complexity and cost. In this talk, more details regarding the system architecture, its experimental test bed and results will be discussed in detail.

\section{Future work}

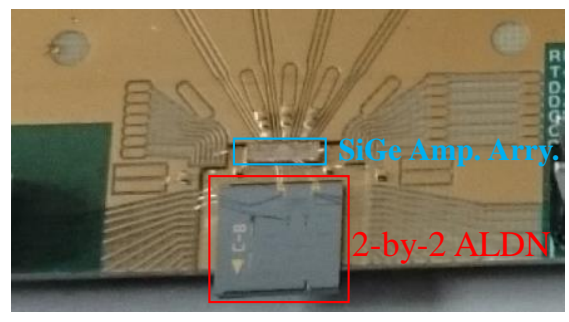

(a) Module of 2-by-2 beamformer at $40 \mathrm{GHz}$
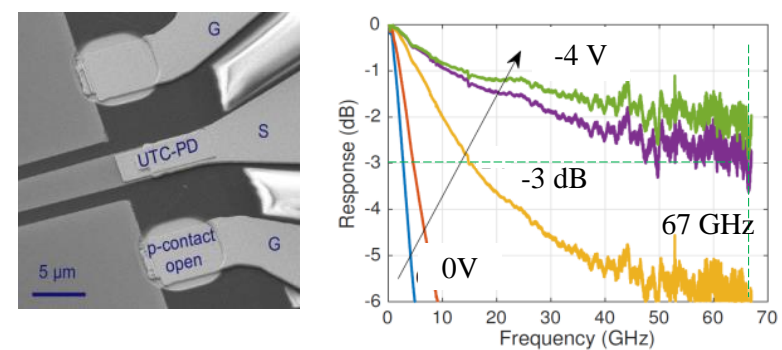

(b) Photo and bandwidth of the UTC-PD

Fig.3. The on-going research on integrated beam-former. (a) full beam steering module with photodiode and electrical amplifier; (b) high-speed uni-travelling carrier photodiode (UTC-PD) on advanced InP membrane platform. .

The broadband mm-wave beam-former based on OTTD is the key component for mm-wave beam steered indoor fiber wireless system. We are currently working on two major research activities concerning the broadband OTTDbased integrated radio beam-former. One is to build up a fully functional optical mm-wave beam steering module including high-speed photodiode and electrical amplifier. The other is to implement the beam-former concept in a more advanced integration platform. As shown in Fig. 3 (a), the 2-by-2 interleaved ALDN is realized in an InP integrated circuit with high-speed photodiodes. The high-speed SiGe amplifier array is co-packaged in a high frequency board with four output connectors. This module provides $40 \mathrm{GHz}$ mm-wave beam steering. As shown in Fig. 3 (b), a uni-travelling carrier photodiode with $>67 \mathrm{GHz}$ bandwidth are realized in our InP membrane platform [11]. The membrane platform brings a lot of benefits for high-performance and compact realization of mm-wave beam-former. More details will be presented in this talk.

\section{Conclusion}

In this talk, we introduce the operation principle of the integrated ALDN. Later, the characterization of the fabricated 1-by-2 ALDN is presented. The indoor fiber wireless networks enabled by the ALDN are elaborated to address different scenarios. The different implementations of the networks are discussed in physical layer. The future work regarding fully functional optical mm-wave beam steering module and the realization of the ALDN concept on our InP membrane platform are discussed as well.

\section{References}

[1] Q. C. Li, N. Huaning, A. T. Papathanassiou and W. Geng, "5G Network Capacity: Key Elements and Technologies," Vehicular Technology Magazine, IEEE 9, 71-78 (2014).

[2] W. Yi, L. Jian, H. Lei, J. Yao, A. Georgakopoulos and P. Demestichas, "5G Mobile: Spectrum Broadening to Higher-Frequency Bands to Support High Data Rates," Vehicular Technology Magazine, IEEE 9, 39-46 (2014).

[3] W. Roh, S. Ji-Yun, P. Jeongho, L. Byunghwan, L. Jaekon, K. Yungsoo, C. Jaeweon, C. Kyungwhoon and F. Aryanfar, "Millimeter-wave beamforming as an enabling technology for $5 \mathrm{G}$ cellular communications: theoretical feasibility and prototype results," Communications Magazine, IEEE 52, 106-113 (2014).

[4] W. Roh, J. Y. Seol, J. Park, B. Lee, J. Lee, Y. Kim, J. Cho, K. Cheun and F. Aryanfar, "Millimeter-wave beamforming as an enabling technology for 5G cellular communications: theoretical feasibility and prototype results," Communications Magazine, IEEE 52, 106-113 (2014).

[5] T. Koonen and C. ZiZheng, "Optically controlled 2D radio beam steering system," in Microwave Photonics (MWP) and the 2014 9th AsiaPacific Microwave Photonics Conference (APMP), 2014 International Topical Meeting on, 2014), 389-391.

[6] O. Raz, S. Barzilay, R. Rotman and M. Tur, "Submicrosecond Scan-Angle Switching Photonic Beamformer With Flat RF Response in the C and X Bands," Lightwave Technology, Journal of 26, 2774-2781 (2008).

[7] B. Vidal, T. Mengual, C. Ibanez-Lopez and J. Marti, "Optical Beamforming Network Based on Fiber-Optical Delay Lines and Spatial Light Modulators for Large Antenna Arrays," Photonics Technology Letters, IEEE 18, 2590-2592 (2006).

[8] Z. Cao, F. Li, A. Reniers, C. W. Oh, H. van den Boom, E. Tangdiongga and A. M. J. Koonen, "Spatial Filtering in a Broadband In-Home OFDM Radio-Over-Fibre Network," Photonics Technology Letters, IEEE PP, 1-1 (2014).

[9] T. P. McKenna, J. A. Nanzer and T. R. Clark, "Photonic Beamsteering of a Millimeter-Wave Array With 10-Gb/s Data Transmission," IEEE Photonics Technology Letters 26, 1407-1410 (2014).

[10] Z. Cao, N. Tessema, S. Latkowski, X. Zhao, Z. Chen, V. Moskalenko, K. A. Williams, H. P. A. van der Boom, E. Tangdiongga and A. M. J. Koonen, "Integrated remotely tunable optical delay line for millimeter-wave beam steering fabricated in an InP generic foundry," Optics letters 40, 3930-3933 (2015).

[11] L. Shen, Y. Jiao, W. Yao, Z. Cao, J. P. van Engelen, G. Roelkens and J. J. G. M. van der Tol, "High-bandwidth uni-traveling carrier waveguide photodetector on an InP-membrane-on-silicon platform." Optics express, 24(8), 8290-8301 (2016). 